\title{
Therapeutic effect of Zeng Ye decoction on primary Sjögren's syndrome via upregulation of aquaporin-1 and aquaporin-5 expression levels
}

\author{
XIAODAN WU ${ }^{1}$, CHANGHONG REN $^{2}$, HONGWEI ZHOU $^{3}$, LIN ZHANG $^{1}$, CHENG JUAN $^{4}$ and YONG YANG ${ }^{1}$ \\ ${ }^{1}$ Department of Herbal Medicine, School of Basic Medical Sciences, Beijing University of Chinese Medicine, Beijing 100029; \\ ${ }^{2}$ Institute of Hypoxia Medicine, Xuanwu Hospital, Capital Medical University, Beijing 100053; \\ ${ }^{3}$ Institute of Basic Research in Clinical Medicine, China Academy of Chinese Medical Sciences, Beijing 100700; \\ ${ }^{4}$ Department of Pathology, Dongzhimen Hospital Affiliated to Beijing University of Chinese Medicine, \\ Beijing 101121, P.R. China
}

Received September 21, 2013; Accepted March 21, 2014

DOI: $10.3892 / \mathrm{mmr} .2014 .2208$

\begin{abstract}
The aim of this study was to investigate the curative effect of Zeng Ye decoction on primary Sjögren's syndrome (pSS) and further explore its underlying mechanism involving aquaporin (AQP)-1 and AQP-5. The pSS model was established based on the immune induction method, and the saliva flow, submandibular gland index, morphological structures of salivary glands, and AQP-1 and AQP-5 protein expression levels in the salivary glands were determined. The saliva flow and the submandibular gland index were significantly reduced in the model group $(\mathrm{P}<0.01$, compared with those in the control group), and significantly increased following interferon (IFN), Zeng Ye decoction extraction (ZYE) and Zeng Ye decoction $(Z Y)$ treatment $(\mathrm{P}<0.01$, compared with those of the model group). Submandibular gland atrophy, fibrous tissue hyperplasia and multiple focal lymphocytic infiltration were observed in the model group and were attenuated when subjected to IFN, ZYE and ZY treatment. The AQP-1 and AQP-5 expression levels increased following IFN, ZYE and ZY treatment $(\mathrm{P}<0.01$, compared with those of the model group), particularly in the ZYE-35 group. This result indicated that ZYE had a significant protective effect on pSS via upregulation of the expression levels of AQP-1 and/or AQP-5. However, the AQP-1 expression levels increased and the AQP-5 expression levels decreased in the model groups compared with those in the control group, which indicated different regulatory
\end{abstract}

Correspondence to: Dr Yong Yang, Department of Herbal Medicine, School of Basic Medical Sciences, Beijing University of Chinese Medicine, No. 11 Beisanhuan Donglu, Chaoyang, Beijing 100029, P.R. China

E-mail: yymark@aliyun.com

Key words: primary Sjögren's syndrome, Zeng Ye decoction, Zeng Ye decoction extraction, aquaporin-1, aquaporin-5, salivary gland, protection, mechanism pathways of the salivary gland damage on the basis of AQP-1 and AQP-5. This study provided a significant reference for the prevention and treatment of $\mathrm{pSS}$.

\section{Introduction}

Sjogren's syndrome (SS) is a multifaceted, chronic and systemic autoimmune disease, which predominantly occurs in females (1-4). The disease includes two processes: Primary SS (pSS) and secondary SS (sSS). pSS occurs in individuals with no other types of rheumatic disease $(5,6)$ and $\mathrm{sSS}$ occurs in individuals who have another type of rheumatic disease, which is most often systemic lupus erythematosus or rheumatoid arthritis (7-9). SS predominantly affects the salivary and lacrimal glands and further results in dry eyes and, in particular, dry mouth (10-12). The damage to the salivary glands in SS cannot be reversed, but the symptoms are controllable. The curative effect of type-I interferon (IFN) treatment in SS has been well-documented (13-15) and thus, it is used as a positive control in the present study.

Zeng Ye decoction is extracted from figwort, Ophiopogon japonicus and Rehmannia glutinosa Libosch. In the field of traditional Chinese medicine (TCM), Zeng Ye decoction, as an important Chinese medicinal agent, has been widely used for relieving constipation due to body fluid deficiency, the condition which is figuratively described as "boat stranding with water depletion' (16). The theory of 'increasing body fluid for curing constipation' was proposed by the famous medical scholar JuTong Wu with regard to epidemic febrile diseases (17). However, the protective effect of Zeng Ye decoction in pSS and its underlying mechanism has not previously been reported to the best of our knowledge. Therefore, the present study aimed to investigate the therapeutic effect of Zeng Ye decoction on pSS and further explore its underlying mechanism.

\section{Materials and methods}

Experimental animals and groups. A total of 48 female specific pathogen-free mice (age, 8-10 weeks; weight, 
18-20 g; Vital River Laboratory Animal Technology Co. Ltd., Beijing, China) were randomly divided into eight groups $(n=6)$, including the control, adjuvant, model, IFN, Zeng Ye docuction extraction treatment after 50 days pSS (ZYE-50), Zeng Ye decoction treatment after 50 days pSS (ZY-50), Zeng Ye decoction extraction treatment after 35 days pSS (ZYE-35), and Zeng Ye decoction treatment after 35 days pSS (ZY-35) groups. This experiment was approved by the Ethics Committee of Beijing University of Chinese Medicine (Beijing, China). The Zeng Ye decoction consisted of $30 \mathrm{~g}$ figwort (Xuan Shen), $24 \mathrm{~g}$ Ophiopogon japonicas (Mai Dong), and 24 g Rehmannia glutinosa Libosch (Sheng Di) (Beijing Tongrentang Co., Ltd., Beijing, China), decocted for $30 \mathrm{~min}$ to produce the solution of $1 \mathrm{~g}$ raw herbs per $1 \mathrm{ml}$ decoction. To obtain the extracting solution of Zeng Ye decoction, the central composite design-response surface methodology and high-performance liquid chromatography were performed to extract and determine the active ingredients from three herbs (Ruscogenin from Mai Dong, Catalpol from Sheng Di, Harpagide and Harpagoside from Xuan Shen). These ingredients were then mixed and adjusted to produce a solution of $1 \mathrm{~g}$ raw herbs per $1 \mathrm{ml}$.

pSS model construction. The aforementioned mice were multi-point injected with pertussis vaccine (batch number: 20111258-2; Wuhan Institute of Biological Products Co., Ltd., Wuhan, China), complete Freund's adjuvant (CFA) and submandibular gland antigen $(200 \mu \mathrm{g} / \mathrm{ml}$; volume: $0.5 \mathrm{ml})$ in the footpad and subcutaneously, and intraperitoneally injected with $0.05 \mathrm{ml}$ pertussis vaccine. The control group did not receive the treatment and the adjuvant group was only injected with CFA ( $0.1 \mathrm{ml} /$ footpad). One and seven days after the first immunization, the immunization was boosted with intraperi-toneal injection of $2.9 \times 10^{10}$ Bordetella pertussis to each group with the exception of the control group. After 21 days from the first immunization, further booster immunizations were performed every 10 days in the model group and treatment group, which involved intraperitoneal injection of $2.9 \times 10^{10}$ Bordetella pertussis and dorsal subcutaneous multipoint injection of emulsified antigen. The adjuvant group were subjected to a further booster immunization with dorsal subcutaneous injection of CFA. Thirty-five and 50 days later, the ZYE-35, ZY-35, ZYE-50 and ZY-50 groups were lavaged with $0.8 \mathrm{ml} / 10 \mathrm{~g}$ Zeng Ye decoction, the control group were lavaged with an equal volume double-distilled $\mathrm{H}_{2} \mathrm{O}$ and the IFN group was intraperitoneally injected with IFN $\alpha-2 b$ (batch number: 20110571; Beijing Kawin Technology Share-Holding Co., Ltd., Beijing, China). Subsequently, the mice were sacrificed with cervical dislocation, the salivary gland was removed for sectioning, and the total protein was extracted to determine the aquaporin (AQP)-1 and AQP-5 expression levels.

Hematoxylin and eosin staining. The slides were deparaffinized and rehydrated, and the sections were marginally over-stained with hematoxylin $(5 \mathrm{~min})$ and fixed, and then excessive stain was removed with tap water. The slides were differentiated and destained for a few seconds in acidic alcohol until they appeared red (4-5 dips). The sections were briefly rinsed in tap water to remove the acid and stained blue in bicarbonate until the nuclei stood out sharply ( 2 min).
The sections were rinsed under running tap water for $8 \mathrm{~min}$, dehydrated and cleaned, or stained with eosin. Hematoxylin stained slides from the last tap water rinse were placed in $70 \%$ ethanol for $3 \mathrm{~min}$. The slides were placed in eosin for $2 \mathrm{~min}$, taken through three changes of $95 \%$ ethanol for $5 \mathrm{~min}$ and then transferred to the first absolute ethanol of the clearing series. The images were captured and analyzed by ImageJ software, version 1.47 (http://rsb.info.nih.gov/ij/download. html).

Immunohistochemical assay. The slides were deparaffinized, rehydrated and washed three times with phosphate-buffered saline (PBS; $5 \mathrm{~min} /$ time). Endogenous peroxidase was inactivated by incubating the sections with $3 \% \mathrm{H}_{2} \mathrm{O}_{2}$ for $30 \mathrm{~min}$. The sections underwent sequential incubations in $10 \%$ normal goat serum in PBS for $30 \mathrm{~min}$ at room temperature. Subsequently, the sections were incubated in mouse monoclonal anti-AQP-1 and AQP-5 (1:100; Abcam, Cambridge, UK) in PBS containing $0.3 \%$ Triton X-100 at $4^{\circ} \mathrm{C}$ overnight. After washing three times for 5 min with PBS, the sections were incubated in peroxidase-conjugated goat anti-mouse IgG (1:200; Zymed Laboratories, Carlsbad, CA, USA) for $1 \mathrm{~h}$ at room temperature. The sections were developed with diaminobenzidine (Sigma-Aldrich, St. Louis, MO, USA) in Tris-buffered saline containing $0.001 \% \mathrm{H}_{2} \mathrm{O}_{2}$ for $30-50 \mathrm{~min}$. The number of positive cells was measured by Image-Pro Plus software, version 7.0 (http://www.mediacy.com/index. aspx?page=IP_Premier) and analyzed by Origin software, version 9.0 (http://www.originlab.com/index.aspx?go= Products/OriginPro).

Western blot assay. The total protein of each group was extracted and quantified, and $35 \mathrm{mg}$ protein was separated by $12.5 \%$ SDS-PAGE. The separated protein was transferred to polyvinylidene difluoride membranes, and then incubated overnight with rabbit anti-mouse AQP-1 antibody (1:500; Abcam) and mouse anti-mouse AQP-5 antibody (1:500; Abcam). The blotted membranes were incubated for $2.5 \mathrm{~h}$ with horseradish peroxidase-labeled goat anti-rabbit secondary antibody (1:1,000; Santa Cruz Biotechnology, Inc., Santa Cruz, CA, USA). Subsequently, the protein bands were read with an electronic scanner and analyzed with the Image-Pro Plus software, version 7.0.

Statistical analysis. All data are expressed as the mean \pm standard deviation. Statistical analysis of the morphometrical quantification of the AQP-1 and AQP-5 positive cells was performed by means of the one-way analysis of variance test. Scheffe's test for group mean comparisons was used when two means were compared. $\mathrm{P}<0.05$ and $\mathrm{P}<0.01$ were considered to indicate a statistically significant difference.

\section{Results}

Construction of the pSS model. The saliva flow and the submandibular gland index were reduced in the model group compared with those in the control group and increased in the IFN, ZYE-50, ZY-50, ZYE-35 and ZY-35 groups compared with those in the model group $(\mathrm{P}<0.01$; Fig. 1$)$. This result indicated that the pSS model was correctly constructed, and 
A

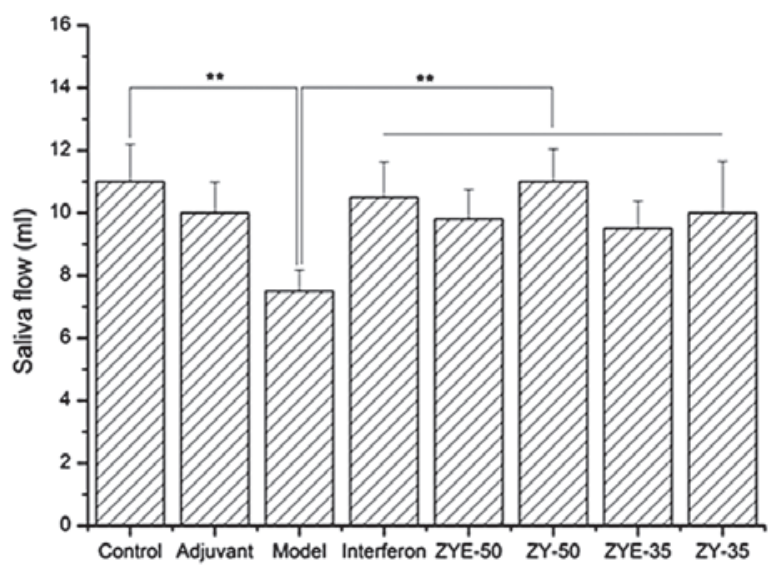

B

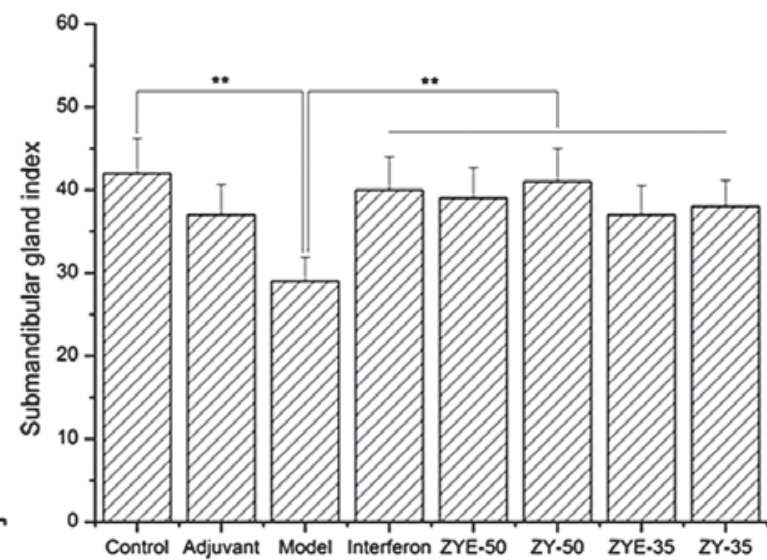

Figure 1. Histograms displaying the salivary gland flow and submandibular gland index. A histogram of (A) the salivary gland flow analysis and (B) the submandibular gland index analysis. The graphs indicated that Zeng Ye decoction increases the saliva flow and submandibular gland index. ${ }^{* *} \mathrm{P}<0.01$. ZYE, Zeng Ye docuction extraction; ZY, Zeng Ye docuction.
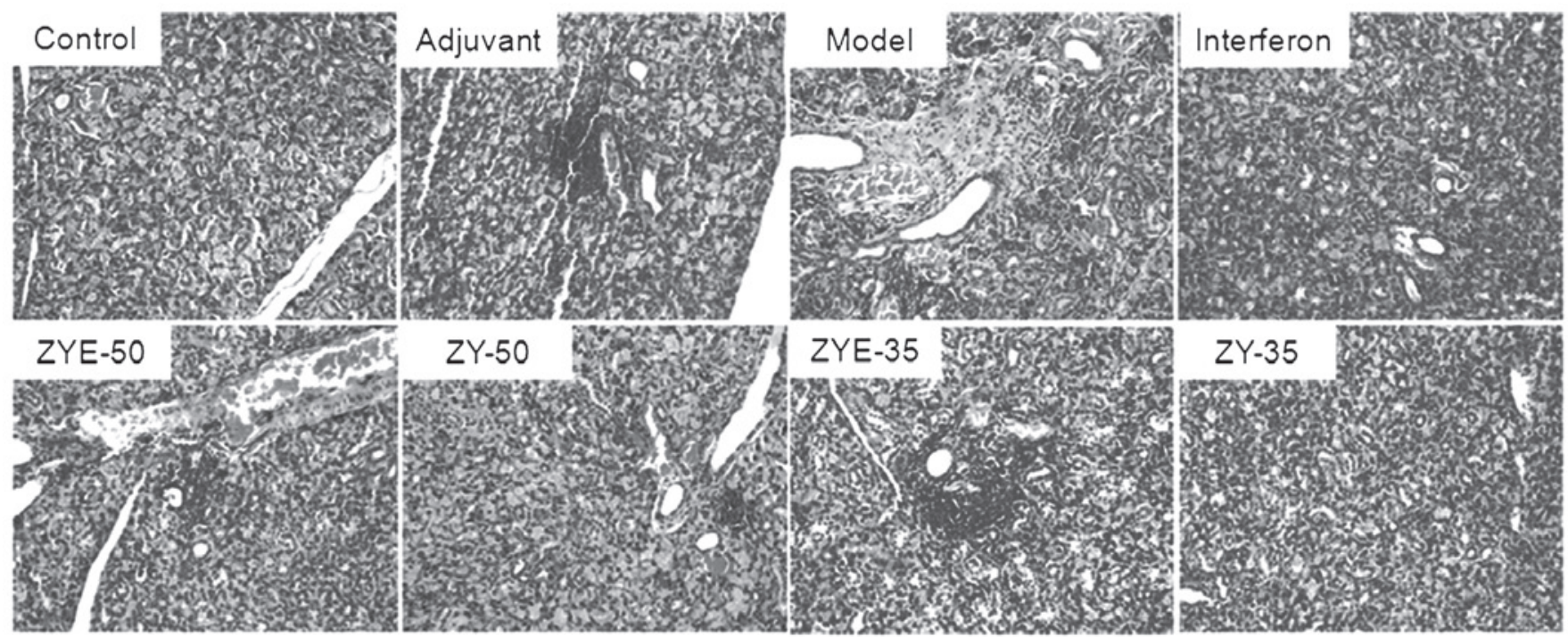

$(200 \times)$

Figure 2. Morphological structure assay of the salivary glands by hematoxylin and eosin staining. The assay indicates that interferon and Zeng Ye decoction treatment effectively alleviate pSS damage $\left({ }^{* *} \mathrm{P}<0.01\right.$, compared with that of the model group). ZYE, Zeng Ye docuction extraction; ZY, Zeng Ye docuction.

that Zeng Ye decoction facilitated the secretion of saliva and recovered the submandibular gland index.

Zeng Ye decoction alleviates the salivary gland damage after pSS, particularly in the ZYE-35 and ZYE-50 groups. The number of stained cells in the salivary gland was increased after the induction of pSS and the damage was effectively alleviated following IFN, ZYE and ZY treatment (Fig. 2). Submandibular gland atrophy, fibrous tissue hyperplasia and multiple focal lymphocytic infiltration were observed in the model group and were recovered when the mice were subjected to IFN, ZYE and ZY treatment.

Zeng Ye decoction increases $A Q P-1$ and $A Q P-5$ expression levels after pSS induction, particularly in the ZYE-35 group. The AQP-1 expression levels were elevated in the model group compared with those in the control group $(\mathrm{P}<0.01)$, and the AQP-1 expression levels were further increased when subjected to ZYE and ZY treatment. Furthermore, the number of cells positive for AQP-1 was significantly increased in the ZYE-35 group compared with that in the ZY-35 (Fig. 3; P<0.01). The AQP-5 expression levels were reduced in the model group compared with those in the control group $(\mathrm{P}<0.01)$, and the AQP-5 expression levels were elevated following the Zeng Ye decoction treatment. Furthermore, the number of cells positive for AQP-5 was significantly increased in the ZYE-35 group compared with that in the ZY-35 group (Fig. 4; $\mathrm{P}<0.01$ ).

Zeng Ye decoction increases $A Q P-1$ and AQP-5 protein expression levels in $p S S$. The western blot analysis results demonstrated that the AQP-1 expression levels were increased 

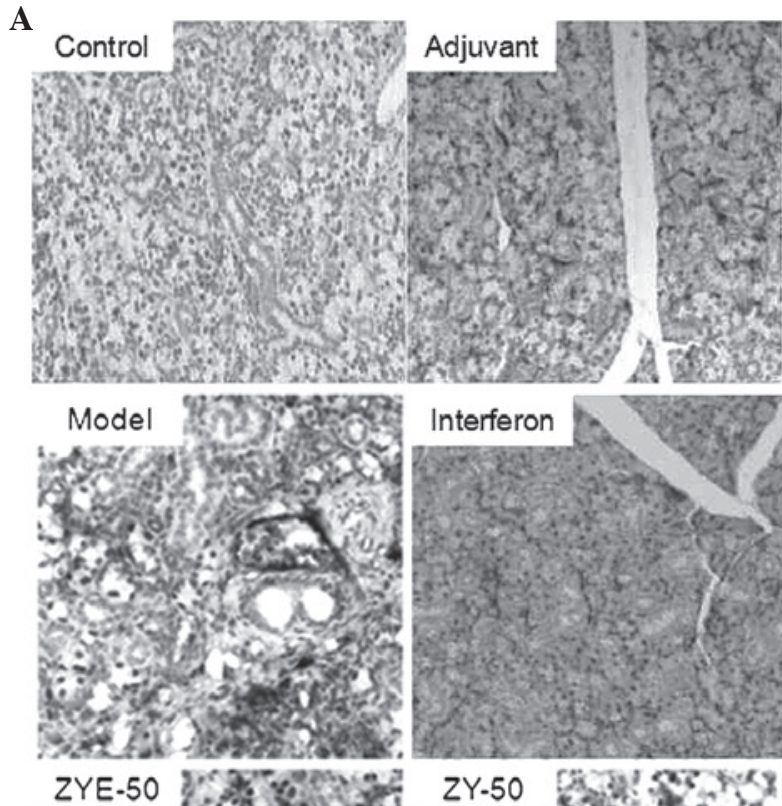

BSE

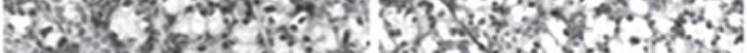

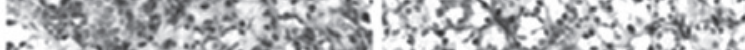

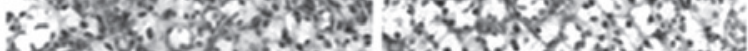

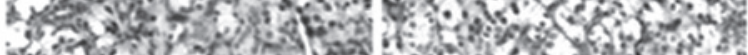

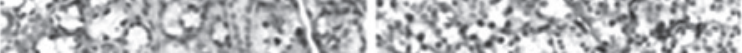

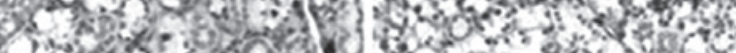
37 6 (3)

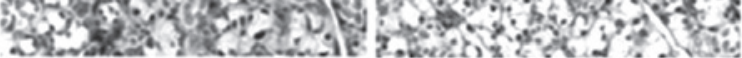

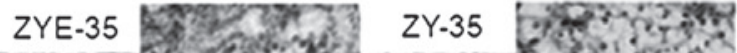

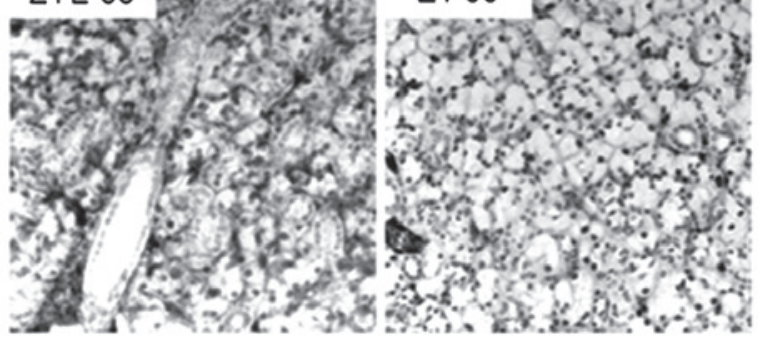
$(400 \times)$

B

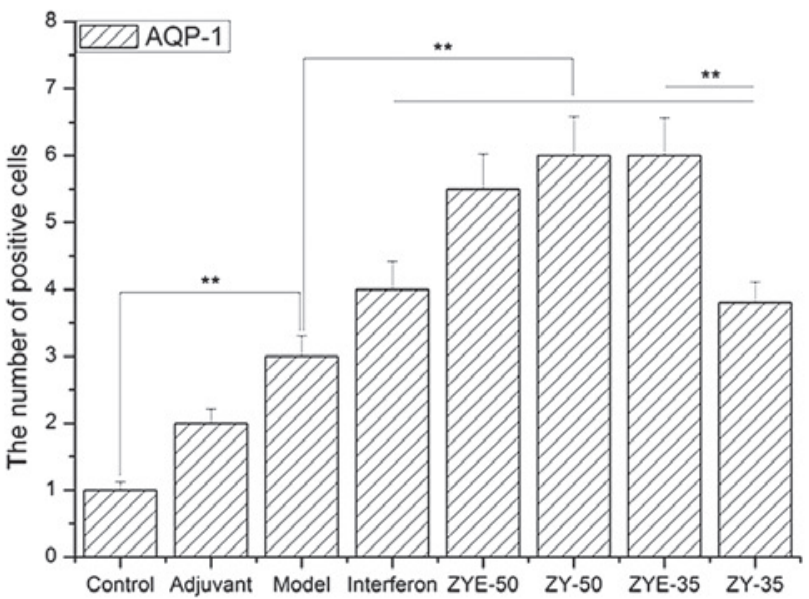

Figure 3. AQP-1 expression level assay in the salivary glands by immunohistochemistry and histogram analysis. (A) Immunohistochemical assay and (B) histogram analysis of the AQP-1 expression levels in the salivary glands The results indicated that the AQP-1 expression levels were elevated when subjected to ZYE and ZY, particularly in the ZYE-35 group ( $\left.{ }^{* *} \mathrm{P}<0.01\right)$. ZYE, Zeng Ye docuction extraction; ZY, Zeng Ye docuction; AQP, aquaporin.
A

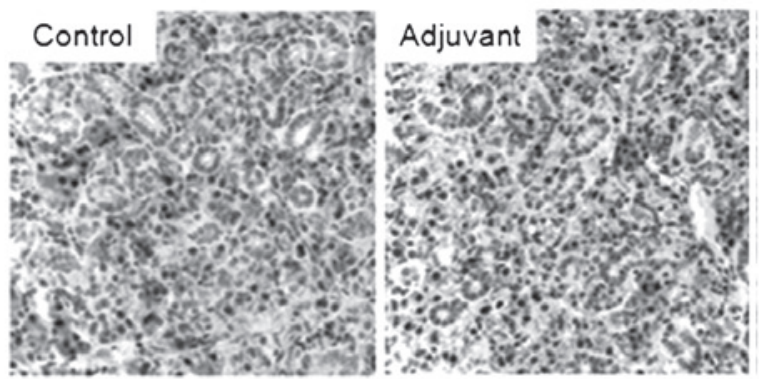

Model erring

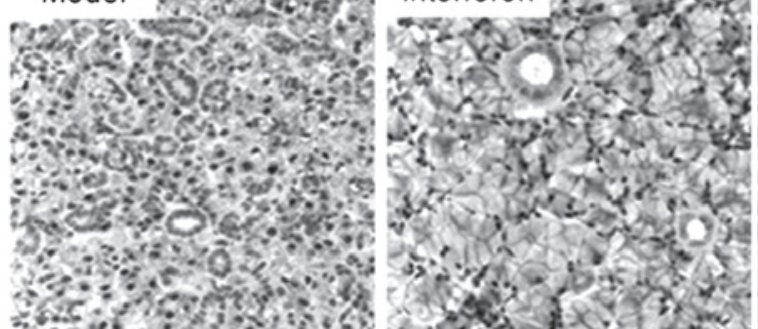

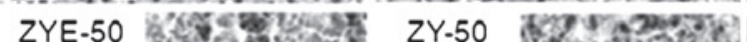

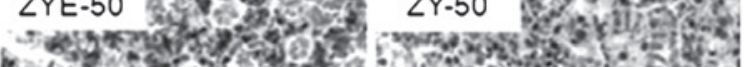

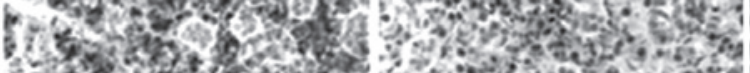

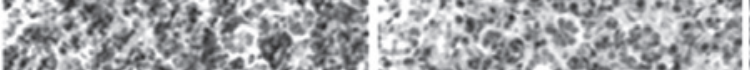

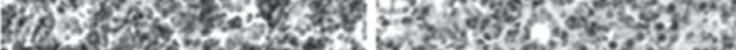

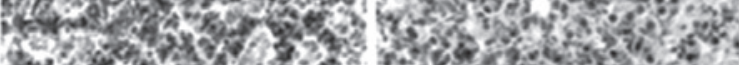

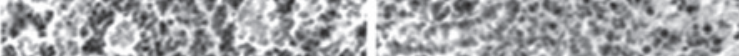
(1) 7 . 2 2 .

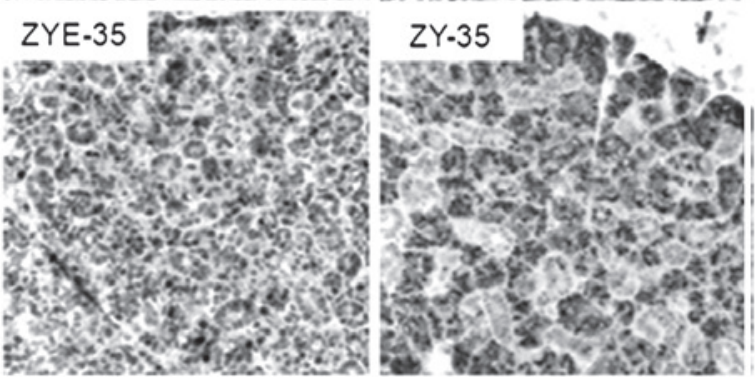

$(400 \times)$

B

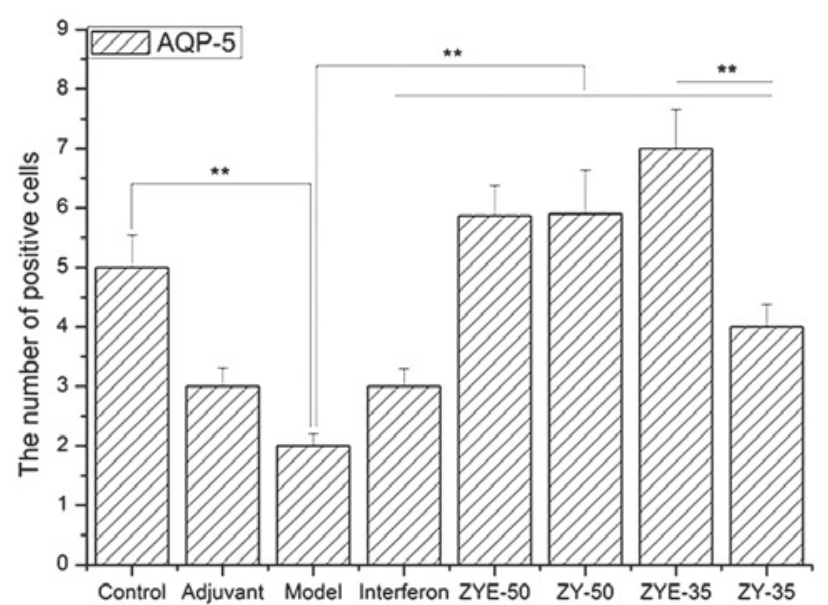

Figure 4. AQP-5 expression level assay in the salivary glands by immunohistochemistry and histogram analysis. (A) Immunohistochemical assay and (B) histogram analysis of the AQP-5 expression levels in the salivary glands. The results indicated that the AQP-5 expression levels were elevated when subjected to ZYE and ZY, particularly in the ZYE-35 group $\left({ }^{* * *} \mathrm{P}<0.01\right)$. ZYE, Zeng Ye docuction extraction; ZY, Zeng Ye docuction; AQP, aquaporin. 
A

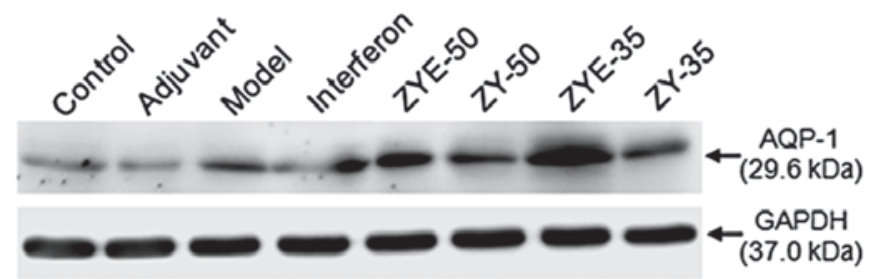

C

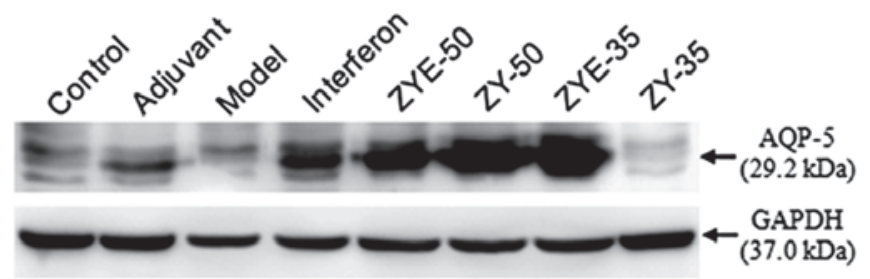

B
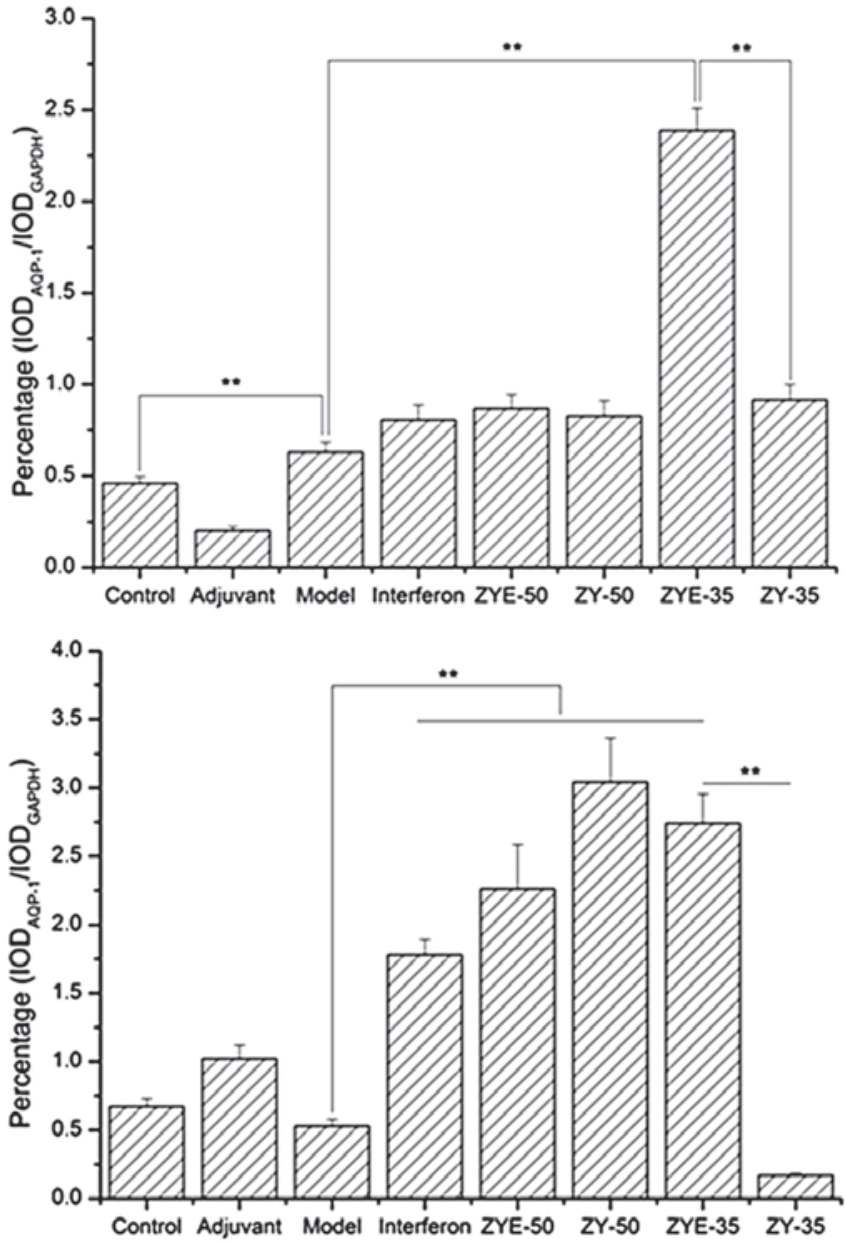

Figure 5. AQP-1 and AQP-5 expression levels assay in the salivary glands by western blot and histogram analysis. The AQP-1 expression levels assay in the salivary glands by (A) western blot and (B) histogram analysis. The AQP-5 expression levels assay in the salivary glands by (C) western blot and (D) histogram analysis. The results indicate that the AQP-1 and AQP-5 expression levels were increased when subjected to ZYE and ZY, particularly the ZYE-35 ("P $<0.01$, compared with those of the model, ZY-35, ZYE-50 and ZY-50 groups). ZYE, Zeng Ye docuction extraction; ZY, Zeng Ye docuction; AQP, aquaporin.

in the model group compared with those in the control and further increased in the IFN, ZYE-50, ZY-50, ZYE-35 and ZY-35 groups, particularly in the ZYE-35 group (Fig. 5A; $\mathrm{P}<0.01$, compared with those of the model group). The AQP-5 expression levels were reduced in the model group and increased in the ZYE-50,ZYE-35 and ZY-50 groups compared with those in the control group. However, the AQP-5 expression levels were significantly reduced in the ZY-35 group, and indicated that ZYE facilitated the recovery of salivary gland damage via upregulation of the expression levels of AQP proteins and further alleviated the inflammatory response (Fig. 5A; $\mathrm{P}<0.01$, compared with those in the $\mathrm{ZY}$ group).

\section{Discussion}

The present study demonstrated that Zeng Ye decoction, a TCM, had a significant curative effect on pSS via upregulation of the levels of AQP-1 and AQP-5. The high-yield of these water channel proteins in the salivary glands facilitated the secretion of fluid, and was beneficial to the recovery from pSS. These results indicated that AQP-1 and AQP-5 may be novel targets in pSS and may provide a significant reference for the prevention and treatment of pSS.
SS is a chronic, multifaceted, high incidence, female-prone autoimmune disease, which specifically targets the salivary and lacrimal glands. The disease results in dry mouth and dry eyes, and further leads to multiple organ and tissue damage $(9,18-20)$. The damage to the salivary glands in SS is severe, although several approaches are used to alleviate dry mouth in the clinic, including drinking water (21-24), chewing gum (25), using saliva substitutes (26) and using prescription medications $(27,28)$. However, there are only methods to relieve the symptoms of the disease, none of which are curative, particularly in pSS. Therefore, the aim of the present study was to investigate the curative effect of Zeng Ye decoction on pSS and further study its underlying mechanism.

Zeng Ye decoction and its extraction had a significant curative effect on $\mathrm{pSS}$ in the present study via upregulation of AQP-1 and AQP-5 expression levels. The AQP-1 expression levels were upregulated and the AQP-5 expression levels were downregulated in the model group compared with those in the control group. This result indicated that SS had different regulatory patterns of AQP-1 and AQP-5. The different patterns may be associated with their different regulatory signaling pathways. Sabapathy (29) demonstrated that AQP-1 only regulated the JNK signaling pathway, and that AQP-5 is mainly involved in 
the nuclear factor- $\kappa \mathrm{B}$ and/or p-c-Jun/c-Fos crosstalk signaling pathway in $\mathrm{C} 3 \mathrm{H} / \mathrm{HeN}$ mice following treatment with lipopolysaccharide and its inhibitor. Therefore, our future studies will further explore the regulatory signaling pathway of AQP1 and AQP5 in the pSS mouse model with Zeng Ye decoction.

In the present study, the different preparation techniques of Zeng Ye decoction, including extraction and water decoction, were selected to separate its effective constituent. The protective effect of the Zeng Ye decoction via different preparation techniques was consistent at 50 days of pSS. At 35 days of pSS, the AQP-1 and AQP-5 expression levels were significantly different between the extraction and water decoction groups. This result may be associated with the different immune mechanisms of the different ingredients of Zeng Ye decoction in the early development of pSS. Our future studies will also explore the detailed protective mechanisms of Zeng Ye decoction on the basis of AQP-1 and AQP-5.

Therefore, the present study demonstrated that Zeng Ye decoction had a significant curative effect in pSS. Zeng Ye decoction significantly upregulated the AQP-1 and AQP-5 expression levels via different mechanisms. This study provided a novel insight into a potential curative treatment for pSS and explored the implied possible mechanism of Zeng Ye decoction.

\section{Acknowledgements}

This study was supported by the National Natural Science Foundation of China (grant nos. 81001495 and 81370090).

\section{References}

1. Fei YY, Li XM, Lin DF, Li MT, Zhang W, et al: Importance of salivary gland focus score in the diagnosis of Sjögren's syndrome. Zhonghua Yi Xue Za Zhi 93: 976-979, 2013 (In Chinese).

2. Susić G, Stojanović R, Milić V, Boricić I, Mandić B and Milenković S: Juvenile Sjögren's syndrome: case report. Srp Arh Celok Lek 141: 228-231, 2013 (In Serbian).

3. Igoe A and Scofield RH: Autoimmunity and infection in Sjögren's syndrome. Curr Opin Rheumatol 25: 480-487, 2013.

4. Cermak JM, Papas AS, Sullivan RM, Dana MR and Sullivan DA: Nutrient intake in women with primary and secondary Sjögren's syndrome. Eur J Clin Nutr 57: 328-334, 2003.

5. Nocturne G and Mariette X: Advances in understanding the pathogenesis of primary Sjögren's syndrome. Nat Rev Rheumatol 9: 544-556, 2013.

6. Routsias JG, Goules JD, Charalampakis G, Tzima S, Papageorgiou A and Voulgarelis M: Malignant lymphoma in primary Sjögren's syndrome: an update on the pathogenesis and treatment. Semin Arthritis Rheum 43: 178-186, 2013.

7. Antero DC, Parra AG, Miyazaki FH, Gehlen M and Skare TL: Secondary Sjögren's syndrome and disease activity of rheumatoid arthritis. Rev Assoc Med Bras 57: 319-322, 2011.

8. Kovács L, Szodoray P and Kiss E: Secondary tumours in Sjögren's syndrome. Autoimmun Rev 9: 203-206, 2010.

9. Baurmash HD: Diagnosing primary and secondary Sjögren's syndrome. J Oral Maxillofac Surg 62: 764-766, 2004.
10. Porola P, Mackiewicz Z, Laine M, Baretto G, Stegaev V, et al: Laminin isoform profiles in salivary glands in Sjögren's syndrome. Adv Clin Chem 55: 35-59, 2011.

11. Busamia B, Gonzalez-Moles MA, Ruiz-Avila I, Brunotto M, Gil-Montoya JA, et al: Cell apoptosis and proliferation in salivary glands of Sjögren's syndrome. J Oral Pathol Med 40: 721-725, 2011.

12. Porola P, Laine M, Virtanen I, Pöllänen R, Przybyla BD and Konttinen YT: Androgens and integrins in salivary glands in Sjögren's syndrome. J Rheumatol 37: 1181-1187, 2010.

13. Szczerba BM, Rybakowska PD, Dey P, Payerhin KM, Peck $\mathrm{AB}$, et al: Type I interferon receptor deficiency prevents murine Sjögren's syndrome. J Dent Res 92: 444-449, 2013.

14. Nordmark G, Eloranta ML and Ronnblom L: Primary Sjögren's syndrome and the type I interferon system. Curr Pharm Biotechnol 13: 2054-2062, 2012.

15. Mavragani CP and Crow MK: Activation of the type I interferon pathway in primary Sjögren's syndrome. J Autoimmun 35: 225-231, 2010

16. Feng Y, Liu Z, Peng Y, Zhang L, Ju P, et al: Validated LC-MS method for simultaneous quantitation of catalpol and harpagide in rat plasma: application to a comparative pharmacokinetic study in normal and diabetic rats after oral administration of Zeng-Ye-Decoction. Biomed Chromatogr 27: 1503-1510, 2013.

17. Tang W: Analysis of Warm Diseases. Beijing People's Medical Publishing House, Beijing, p64, 1963 (In Chinese).

18. Primary and secondary Sjögren's syndrome. Lancet 2: 730-731, 1984.

19. Amador-Patarroyo MJ, Arbelaez JG, Mantilla RD, Rodriguez-Rodriguez A, Cárdenas-Roldán J, et al: Sjögren's syndrome at the crossroad of polyautoimmunity. J Autoimmun 39: 199-205, 2012

20. Baldini C, Talarico R, Tzioufas AG and Bombardieri S: Classification criteria for Sjögren's syndrome: a critical review. J Autoimmun 39: 9-14, 2012.

21. Tanner K, Roy N, Merrill RM, Kendall K, Miller KL, et al: Comparing nebulized water versus saline after laryngeal desiccation challenge in Sjögren's Syndrome. Laryngoscope 123: 2787-2792, 2013

22. Castro I, Sepulveda D, Cortés J, Quest AF, Barrera MJ, et al: Oral dryness in Sjögren's syndrome patients. Not just a question of water. Autoimmun Rev 12: 567-574, 2013.

23. Wu AJ: The oral component of Sjögren's syndrome: pass the scalpel and check the water. Curr Rheumatol Rep 5: 304-310, 2003.

24. Steinfeld S, Cogan E, King LS, Agre P, Kiss R and Delporte C: Abnormal distribution of aquaporin-5 water channel protein in salivary glands from Sjögren's syndrome patients. Lab Invest 81: $143-148,2001$.

25. Miyawaki S, Torikai K, Natsume I, Nobunaga T, Ohtsuka E, et al: Evaluation of two quantitative tests for salivary secretion - the chewing gum test and the Saxon test in normal subjects and in patients with Sjögren's syndrome. Ryumachi 31: 22-27, 1991 (In Japanese).

26. van der Reijden WA, van der Kwaak H, Vissink A, Veerman EC and Amerongen AV: Treatment of xerostomia with polymer-based saliva substitutes in patients with Sjögren's syndrome. Arthritis Rheum 39: 57-63, 1996.

27. Ramos-Casals M, Brito-Zerón P, Sisó-Almirall A, Bosch X and Tzioufas AG: Topical and systemic medications for the treatment of primary Sjögren's syndrome. Nat Rev Rheumatol 8: 399-411, 2012.

28. Simmons DD, Al-Hashimi I and Haghighat N: Effect of xerostomic medications on stimulated salivary flow rate in patients with Sjögren's syndrome. Quintessence Int 31: 196-200, 2000.

29. Sabapathy K: Role of the JNK pathway in human diseases. Prog Mol Biol Transl Sci 106: 145-169, 2012. 